\title{
Le jeu des arts, sous la direction de Matteo Majorano
}

\section{Chiara Rolla}

\section{(2) OpenEdition}

\section{Journals}

\section{Edizione digitale}

URL: http://journals.openedition.org/studifrancesi/30817

DOI: 10.4000/studifrancesi.30817

ISSN: 2427-5856

\section{Editore}

Rosenberg \& Sellier

\section{Edizione cartacea}

Data di pubblicazione: 1 avril 2006

Paginazione: 193-194

ISSN: 0039-2944

\section{Notizia bibliografica digitale}

Chiara Rolla, «Le jeu des arts, sous la direction de Matteo Majorano», Studi Francesi [Online], 148 (XLX | I) | 2006, online dal 30 novembre 2015, consultato il 18 avril 2021. URL: http://

journals.openedition.org/studifrancesi/30817 ; DOl: https://doi.org/10.4000/studifrancesi.30817 


\title{
Le jeu des arts, sous la direction de Matteo Majorano
}

\author{
Chiara Rolla
}

\section{NOTIZIA}

Le jeu des arts, sous la direction de MATTEO MAJORANO, Bari, Edizioni B.A. Graphis, 2005, pp. 234.

1 Gli studi che il volume raccoglie e i due entretiens che presenta (MILENA MASELLI - TANGUY VIEL, Conversation, pp. 110-120 e JEAN-LuC Defromont - CAmille LAURenS, Entretien, pp. 173-185) riprendono quel dibattito tra letteratura e arti a cui il XX secolo ha dedicato buona parte della sua riflessione critica, concentrandosi qui sulla produzione narrativa francese detta del contemporaneo estremo. MATTEO MAJORANO (Un regard qui change les choses, pp. XVII-XXII), con uno sguardo d'insieme e cogliendo i denominatori comuni che i diversi contributi hanno messo in luce, propende per una lettura "artistica" del presente, dove nulla è distinto dall'arte e dove per ciò stesso "tout est art" (p. XXI).

2 La miscellanea comprende quattro sezioni. Nella prima ("Ouvertures") MATTEO MAJORANO analizza tre romanzi che in maniera del tutto diversa mettono in scena la partecipazione dell'arte alla scrittura e si interroga sul nuovo ruolo del critico letterario, il quale, all'interno della società dei consumi che ha improntato dei suoi meccanismi anche l'universo della letteratura, è chiamato ad esprimersi con tempestività evitando che delle logiche estranee al lavoro artistico impongano il loro orientamento (L'art contre l'art et l'art de chacun, pp. 5-22). PIERRE BERGOUNIOUX riflette sulle conseguenze del capitalismo sulla letteratura mettendo in rilievo il tragico effetto che le logiche del profitto hanno su di essa (Un art à part, pp. 23-28).

«La parole dictée par l'écriture est avant tout un chant de la pensée» (p. 37): così si conclude la riflessione di ALAIN FLEISCHER, punto di arrivo della sua ricerca di un'emancipazione della scrittura dalla mano e dal controllo dell'occhio (Ecrire : la main et la voix, pp. 29-37). 
4 La seconda sezione ("Images") si apre con il contributo di DOMINIQUE VIART: «Sur le motif» è un'espressione che ritorna frequentemente ne La vie de Joseph Roulin di Michon. L’A. qui la analizza sia nel suo significato letterale che in quelli più celati («Sur le motif » : l'image prise au mot, pp. 41-55).

ELISABETTA LONGARI passa in rassegna le più recenti avanguardie pittoriche e coglie gli stretti rapporti che sono intercorsi a partire dagli anni Sessanta tra arti visive e letteratura (Letteratura e scrittura come modelli per le arti visive, pp. 56-66).

Il contributo di GIANFRANCO RUBINo verte su Paléo Circus, il romanzo di Rouaud in cui, parallelamente all'infanzia dell'umanità, viene rintracciata anche la genesi del gesto artistico (Jean Rouaud : les origines de l'art, pp. 67-76), mentre MARIE THÉRÈSE JACQUET indaga sul passaggio dalla scrittura alla scultura in Pierre Bergounioux (Une écriture en fer, pp. 77-95).

7 ROSA GALLI PELLEGRINI ricerca gli echi dei dibattiti filosofici e artistici della rivoluzione culturale del 1968 all'interno dell'Iconoclaste di Nadaud, romanzo che per la sua forma si inscrive all'interno di quel processo di destrutturazione delle strutture narrative iniziato negli anni Sessanta (Déconstruire et reconstruire : L'Iconoclaste d'Alain Nadaud, pp. 96-109).

8 TANGUY VIEL chiude la seconda sezione riflettendo sullo stretto legame che intercorre tra narrativa contemporanea e cinema (La fabrique de l'image, pp. 121-28).

9 La terza parte ("Mouvements") si apre con MARC DAMBRE che dedica il suo contributo a Benoît Duteurtre e alla sua capacità di intersecare, all'interno delle sue opere, la riflessione letteraria con il suo interesse per la musica (Benoit Duteurtre entre fictions, discours et arts : l'histoire en balance, pp. 131-47).

10 JEAN-BERNARD VRAY riflette sulla presenza significativa della canzone all'interno di alcuni romanzi contemporanei francesi (Le roman connaît la chanson, pp. 148-66), mentre CLAUDE ARNAUD si interroga su quale possa essere la forma letteraria meglio capace di rispondere a quel desiderio di affabulazione insito in ogni uomo (Le roman hors $d u$ roman, pp. 167-72).

11 Il coreografo DINO VERGA illustra attraverso il suo lavoro il difficile compito di trasporre in danza un testo letterario (La narratività astratta, pp. 186-90).

12 DOMINIQUE GAULTIER inaugura la quarta ed ultima sezione del volume ("Variations ") con un contributo in cui riemergono gli echi del '68, della contestazione e della rivoluzione culturale (La découverte et la redécouverte d'auteurs, pp. 193-98).

13 ANDREA GESSNER è un giovane editore che ha saputo riconoscere nella narrativa francese contemporanea una grande varietà e ricchezza: con la casa editrice "Nottetempo" (fondata nel 2002) ha tradotto e pubblicato infatti alcuni romanzi contemporanei francesi (Un giovane editore per una letteratura giovane, pp. 199-201).

14 MAURO GIANCASPRO, direttore della Biblioteca Nazionale di Napoli, esamina lo stretto rapporto che da sempre lega la lettura alla scrittura, all'interno del quale, il più delle volte, è la prima a determinare la seconda: la catena del libro è infatti una struttura ad anello, dove la prima e l'ultima maglia finiscono col coincidere (La biblioteca pubblica, fucina di scrittura, pp. 202-08).

15 Chiude la miscellanea il contributo di ROSANNA GAETA. Qui vengono toccati alcuni problemi fondamentali del mercato librario italiano: in prima istanza lo scarso interesse per la lettura dimostrato dalla popolazione e in secondo luogo, ma non certo 
di minore importanza, le logiche commerciali e di profitto a cui gli editori sottomettono le loro scelte (Coltivare lettori, pp. 209-14). 